\title{
Introduction to the special issue: Vision after Premature Birth
}

\author{
Daphne L. McCulloch • Anne B. Fulton
}

Published online: 31 July 2013

(C) Springer-Verlag Berlin Heidelberg 2013

Preterm birth is a major challenge to perinatal health care and is an important risk factor for visual and neurological impairment. In developed countries, the mortality rates of very preterm birth are declining, but the $1-2 \%$ prevalence rates of significant morbidity remain unchanged [1]. Thus, there is a growing population of at-risk survivors. Research to improve understanding, management, and prevention of associated impairment of vision is therefore a priority.

Prematurity can adversely affect the development of structures and function throughout the entire visual system, from the anterior eye to the cortical brain regions serving higher-order visual processing. The aim of this special issue is to present recent research developments that highlight the diverse consequences of premature birth for the visual system.

This issue contains two manuscripts from the research team at Boston Children's Hospital, based on the rat model of retinopathy (ROP) of prematurity. The first, Favazza et al., demonstrates for the first time

D. L. McCulloch ( $\square)$

Vision Sciences, Glasgow Caledonian University, Glasgow, UK

e-mail: D.L.McCulloch@gcu.ac.uk

Present Address:

D. L. McCulloch

School of Optometry and Vision Sciences, University

of Waterloo, Waterloo, ON, Canada

A. B. Fulton

Harvard Medical School, Boston, MA, USA in the rat that pathological vascular changes anterior to the retina, in the tunica vasculosa lentis occur, indicating that the vascular anomalies of prematurity may be widespread. The fundamental processes underlying regulation of vascular growth in prematurity are addressed in their second manuscript. Employing next-generation sequencing analysis, Griffith et al., has identified a number of genes and pathways that are potential novel targets for therapeutic intervention in ROP.

Mactier et al., from Glasgow, present evidence that ERG abnormalities (suppressed oscillatory potentials) in the human preterm retina are physiological forerunners of ROP and may assist in identifying those requiring early intervention.

The Functional Neuroimaging Unit at the Hospital for Sick Children in Toronto has been at the forefront of recent improvements in neuroimaging for preterm infants. In Chau et al., this group presents a review of preterm brain development, focused on the structures subserving vision, and identifies the basis for visual and perceptual sequelae of prematurity. Bosworth et al. present evidence that regressed ROP is associated with long-term reductions in contrast sensitivity functions and highlights the vulnerability of the magnocellular pathways.

Dutton's review of disorders of higher-order visual function highlights the impact and range of functional deficits associated with premature birth. His ongoing clinical research has shown a high rate of such deficits, including prematurely born children who have passed 
through medical and educational assessment and surveillance with their perceptual difficulties going undetected.

Visual sequelae of premature birth are complex and diverse. This special issue provides a snapshot of topical research that we hope will inform and stimulate interest in the field.

\section{Reference}

1. Tucker Janet, McGuire William (2004) Epidemiology of preterm birth. BMJ 329:675 